\title{
LA RESPONSABILIDAD SOCIAL DEL GRUPO EPM: UNA NUEVA POSTURA POLÍTICA FRENTE AL TERRITORIO*
}

Jorge Andrés Polanco López de Mesa**

* doi:10.11144/Javeriana.cao27-49.rsge. Este artículo es producto de la investigación "Lineamientos para la integración de los componentes económico, social y ambiental en el proceso de incorporación de la sostenibilidad en la empresa" financiada por la Vicerrectoría de investigación de la Universidad de Medellín, Medellín, Colombia, de enero hasta diciembre de 2013. El artículo se recibió el 30/08/2014 y se aprobó el 01/12/2014. Sugerencia de citación: Polanco, J. (2014). La responsabilidad social del grupo EPM: una nueva postura política frente al territorio. Cuadernos de Administración, 27 (49), 65-85. http://dx.doi.org/10.11144/Javeriana.cao27-49.rsge

** Doctorado en Socio-economía del desarrollo de la École des Hautes Études en Sciences Sociales de Paris, Francia, 2007. Profesor Asistente adscrito al doctorado en Administración de la Universidad de Medellín, Medellín, Colombia. Correo electrónico: japolanco@udem.edu.co 


\section{La responsabilidad social del grupo EPM: una nueva postura política frente al territorio}

\section{RESUMEN}

El Grupo EPM experimenta un cambio notable en su estrategia de responsabilidad social. El objetivo de este trabajo es analizar la relación entre el cambio de estrategia y la postura política de la empresa en el área de influencia de la hidroeléctrica Río Grande II en Colombia. La responsabilidad social se asume teóricamente como una iniciativa de sostenibilidad corporativa. La metodología de investigación es un estudio de caso exploratorio. Los resultados muestran que la empresa busca una posición más central en su relación con el Estado y la comunidad local. El relacionamiento con el Estado se orienta hacia la construcción colectiva del territorio, y con la comunidad local se busca mayor participación. Esta nueva postura corporativa conlleva a procesos inexplorados donde se compartan costos y beneficios con el Estado y la comunidad.

Palabras clave: Responsabilidad social, sostenibilidad, estrategia corporativa, territorio, energía.

Clasificación JEL: M14, Q56, R11

\section{The group EPM social responsibility: A new political stance against the territory}

\section{Abstract}

The Grupo EPM is experiencing a remarkable change in its strategy of social responsibility. The aim of this paper is to analyses the relationship between the strategy change and the political stance of the company in the hydropower Río Grande II area of influence in Colombia. Social responsibility is assumed theoretically as a corporate sustainability initiative. An exploratory case study is adopted. Results suggest that the corporation seeks a more central position in the relationship with the State and the local community. The relationship with the State is oriented towards the collective construction of territory, and with the local community is desired to be more participatory. This new corporative stance leads to still unexplored processes where costs and benefits are shared with the State and the community.

Keywords: Social responsibility, sustainability, corporate strategy, territory, energy.

JEL Classification: M14, Q56, R11

\section{A responsabilidade social do Grupo EPM: uma nova postura política no território}

\section{RESUMO}

O Grupo EPM experimenta uma notável mudança na sua estratégia de responsabilidade social. O objetivo deste artigo é analisar a relação entre a mudança de estratégia e a posição política da empresa na área de influência da usina hidrelétrica de Río Grande II na Colômbia. A responsabilidade social é assumida em teoria como uma iniciativa de sustentabilidade corporativa. A metodologia da pesquisa é um estudo de caso exploratório. Os resultados mostram que a empresa busca uma posição mais central na sua relação com o Estado e a comunidade local. A relação com o Estado é orientada para a construção coletiva do território, e com a comunidade local espera ser mais participativo. Essa abordagem corporativa leva a processos ainda inexplorados em que os custos e os benefícios são compartilhados com o Estado e a comunidade.

Palavras-chave: Responsabilidade social, sustentabilidade, estratégia corporativa, território, energia.

Classificação JEL: M14, Q56, R11 


\section{Introducción}

La sostenibilidad del negocio de generación de energía hidroeléctrica depende cada vez más del manejo de embalses, pues es allí donde está en juego la disponibilidad y la calidad del recurso hídrico. De acuerdo con múltiples experiencias en el mundo (Wang et al., 2013; UNEP, 2007), es cada vez más aceptada la necesidad de incluir la participación de grupos de interés en el manejo socio-ambiental de las áreas de influencia de estas obras de infraestructura, buscando así la gobernanza del entorno del negocio.

Se trata por ejemplo del manejo del impacto de la generación de energía en las condiciones de vida de la población, en la biodiversidad y en la erosión de suelos. En Colombia, hay dificultades para mejorar las condiciones de vida por la vía normativa, recurriendo a las tasas por uso de agua (Agudelo et al., 2012), y existen procesos de deforestación de las cuencas tributarias que inciden en la sedimentación de los embalses (Polanco, 2012).

Ante estos problemas, el Grupo Empresas Públicas de Medellín (EPM) -la segunda empresa más grande del país en el sector energético después de Ecopetrol- ha diseñado una nueva estrategia de sostenibilidad del negocio de generación de energía, basada en la responsabilidad social empresarial (EPM, 2012). Por medio de un novedoso modelo de relacionamiento con los grupos de interés, este grupo empresarial busca asegurar la perennidad y la calidad del recurso hídrico.

Sin embargo, esta nueva estrategia de sostenibilidad debe enfrentar amenazas del entor- no que parecen ser producto de una lógica de enclave económico que aún prevalece en el manejo de sus proyectos (Agudelo et al., 2012; Polanco, 2011). ¿Cómo define este cambio de estrategia la postura política de la empresa frente al territorio?

Para responder a esta pregunta nos inspiramos en la estrategia empresarial como proceso racional y organizativo (Mintzberg, 2009; Mintzberg et al., 1998). Es decir que el proceso de incorporación de la sostenibilidad en la empresa, mediante la responsabilidad social empresarial, es sobre todo voluntario y parte del conocimiento del entorno pero también considera el aprendizaje como segmento del mismo (Lozano, 2012; March et al., 2011; Garriga y Melé, 2004).

Por eso partimos del supuesto de que el aprendizaje de la empresa subyacente al cambio de estrategia, contribuiría a la sostenibilidad del negocio de generación de energía si se evidencia una mayor centralidad del Grupo EPM en el relacionamiento con los grupos de interés externos.

En este artículo se analiza la evolución de la estrategia de responsabilidad social del Grupo EPM entre 2000 y 2013, para esbozar su relación de poder en el área de influencia de la hidroeléctrica Río Grande II, situada en el departamento de Antioquia, Colombia.

Optamos por un análisis cualitativo y exploratorio, en el sentido de Bernal (2010) y de Bonilla y Rodríguez (1997), porque una primera aproximación inductiva al problema del cambio de estrategia hace partícipe a los grupos de interés. Se elige el método de 
caso según Yin (2009), porque existe poca información y es necesario triangular los datos emergentes de sus testimonios sobre la relación entre la empresa y su entorno.

Este texto inicia con una presentación más amplia de los planteamientos conceptuales y metodológicos sobre sostenibilidad, responsabilidad social, territorio, empresa y estudio de caso exploratorio, para luego examinar los resultados y evocar el aprendizaje latente en la nueva estrategia para la sostenibilidad del negocio de generación de energía. Finalmente, concluimos el trabajo con lo más destacado del cambio y su efecto en el relacionamiento de la empresa con su entorno, y con una propuesta de futuras investigaciones.

\section{Sostenibilidad y responsabilidad social}

La sostenibilidad es una práctica social en la cual confluyen simultáneamente intereses económicos, sociales, ambientales e intergeneracionales, en aras de un estado dinámico ideal de la relación entre el ser humano y su entorno (Lozano, 2008; Robinson, 2004). En esta práctica confluyen el Estado, el ciudadano y la empresa, toda vez que la sostenibilidad se relaciona con esta última a través de la estrategia (Lozano, 2012).

La estrategia empresarial es entendida aquí como la forma, modelo, planeamiento o perspectiva, definida tanto desde arriba como desde abajo, en que la empresa despliega sus recursos en un entorno específico para alcanzar sus objetivos a largo plazo (Mintzberg, 2009; Mintzberg et al., 1998).
Existe un punto de encuentro entre sostenibilidad y estrategia cuando la empresa busca un estado dinámico con su entorno que la haga competitiva en el sentido de Porter y Kramer (2011). Pero esta competitividad no está determinada solamente por la estructura de la industria de producción de energía, sino también, como lo plantean Chen y Miller (2012), por la respuesta de la empresa frente a las especificidades espacio-temporales del mercado. Esto quiere decir que la competitividad es sobre todo dinámica y por tanto puede desaparecer.

De esta manera, la búsqueda de estrategias desde un enfoque racionalista, donde predomina la planificación, no es suficiente para entender la sostenibilidad, dado que la relación entre la empresa y su entorno es también un proceso de adaptación al territorio. A continuación, presentamos primero el territorio como entorno empresarial para luego precisar por qué la responsabilidad social empresarial puede verse como una iniciativa de sostenibilidad corporativa.

\subsection{El territorio visto como entorno empresarial}

El punto de partida para entender la relación entre la sostenibilidad y la estrategia empresarial es el de una lectura territorial del entorno en donde las instituciones juegan un papel preponderante. Es decir, la estrategia de sostenibilidad de una empresa, la vincula directamente con su entorno y, este vínculo está definido por el modelo de desarrollo que se quiera para la sociedad. 
Hoy en día, este desarrollo se busca sostenible en tanto que es un proceso orientado al bienestar de la población actual sin comprometer aquel de las generaciones futuras (Brundtland, 1987). Se trata de un nuevo modelo de desarrollo provisto de un discurso ya bastante globalizado, pero con un camino largo por recorrer en la práctica local (Polanco, 2009).

La práctica local del desarrollo sostenible es un proceso de aprendizaje que debe ser monitoreado y mejorado (OCDE, 2011; Stag1, 2007), en donde participan actores gubernamentales, empresariales y sociales, cuyas relaciones definen reglas de juego que pueden ser formales o informales (North, 1991).

Vistas como instituciones, estas reglas de juego son fundamentales en la práctica del desarrollo sostenible (Dovers y Hezri, 2010; Soderbaum, 2009; Fischer et al., 2007). Al analizar las relaciones de las empresas con otros sectores de la sociedad y el Estado se ha observado que las reglas de juego informales son más efectivas (Perez-Batres et al., 2011). Esto quiere decir que las empresas contribuyen al desarrollo sostenible principalmente de manera voluntaria.

En el caso de las empresas generadoras de energía hidroeléctrica, las reglas de juego se derivan del relacionamiento con otros agentes en el territorio, sobre todo en el primer eslabón de la cadena de valor del negocio, donde la perennidad y la calidad del recurso hídrico son una condición necesaria. Esto quiere decir que, por ejemplo, durante la fase de operación de las hidroeléctricas el manejo de las áreas tributarias de sus embalses estaría relacionado con la revalorización del papel del territorio en el desarrollo económico local: la creación de valor a nivel local no podría desconocer la necesidad de agua de buena calidad para la generación de energía.

Esta revalorización del territorio se plantea desde dos puntos de vista: en primer lugar, en el territorio están los recursos tangibles (agua, infraestructura, etc.) e intangibles (conocimiento, capital social, etc.) que hacen viable una actividad productiva organizada de manera ascendente, es decir, tejida en red desde abajo, para crear valor sobre la base de dichos recursos (Vazquez-Barquero, 2009, 2008; Pecqueur, 2005). En segundo lugar, esta interacción recursos-actividad-organización es posible toda vez que se cuente con la "tecnología política" que plantea Elden (2010): medir los recursos y controlar el territorio por medio de arreglos institucionales.

La sostenibilidad en el entorno de empresas de generación de energía hidroeléctrica necesita reglas de juego que vinculen a los grupos de interés con una lectura territorial de las áreas tributarias de los embalses. Estas reglas de juego fomentan sinergias públicoprivadas propias de la gobernanza (Polanco, 2010; Lockwood, 2010). Sin embargo, la sostenibilidad no es solamente un problema de lectura territorial del entorno, sino también de incorporación en la empresa.

\subsection{La RSE: iniciativa de sostenibilidad corporativa}

El concepto de sostenibilidad corporativa considera tanto el desempeño como la gober- 
nanza de la sostenibilidad. De acuerdo con Schneider y Meins (2012), el desempeño de la sostenibilidad se refiere al enfoque analítico de la triple cuenta, donde el desempeño se mide en tres dimensiones: económica, ambiental (ecológica) y social. Sin embargo, este enfoque no resulta suficiente para dar cuenta del desempeño de la sostenibilidad, dado que se limita a la interacción parcial y estática entre dimensiones (Lozano y Huisingh, 2011). Por tanto, el desempeño de la sostenibilidad es más visible desde el enfoque del Equilibrio Dinámico Ideal en la medida en que se privilegia la interacción total y dinámica entre las dimensiones (Lozano, 2008).

El impacto de la organización en el desempeño de la sostenibilidad depende de cómo se haga operacional o cómo se incorpore en la misma (Lozano, 2012). Es aquí donde la gobernanza de la sostenibilidad entra en juego, entendiéndose como el catalizador del desempeño futuro de la sostenibilidad, es decir, como el potencial que tiene la empresa para el desempeño tanto de la dimensión económica como de las dimensiones ambiental y social (Schneider y Meins, 2012).

Una sola iniciativa para la incorporación de la sostenibilidad no es suficiente y todas ellas deben articularse con el sistema corporativo. Es decir que, iniciativas como la ecología industrial, la contabilidad ambiental y social, la producción más limpia y la responsabilidad social empresarial deben visualizarse en la estrategia y la estructura corporativas (Lozano, 2012).

Pero la gobernanza de la sostenibilidad no es solamente un problema técnico, sino también político. La efectividad del proceso de incorporación de la sostenibilidad en la empresa también depende de la posición ideológica, los intereses y las reglas de juego evidenciados por la corporación (Polanco, 2013a). Desde la perspectiva de la teoría neo-institucional, Matten y Moon (2008) argumentan que esta postura corporativa obedece a qué tan explícita o implícita esté. La una se diferencia de la otra en la medida en que:

Implicit CSR, however, is not conceived of
as a voluntary and deliberate corporate
decision but, rather, as a reaction to, or
reflection of, a corporation's institutional
environment, whereas explicit CSR is the
result of a deliberate, voluntary, and often
strategic (Porter \& Kramer, 2006) decision
of a corporation (Matten y Moon, 2008,
p. 410).

Sin embargo, como lo evidencian PerezBatres et al. (2011), se han observado más efectivas las acciones voluntarias. Por tanto, es la postura corporativa explícita la que parece contribuir a la gobernanza de la sostenibilidad.

En definitiva, cuando se incorpora la sostenibilidad en la empresa, la visión del proceso de formación de la estrategia obedece no sólo a un enfoque racional, sino también organizativo. Es decir que la formación de la estrategia obedece a procesos formales y analíticos, así como también a procesos mentales, de aprendizaje o de poder (Mintzberg, 2009; Mintzberg et al., 1998). Mientras que en el primer grupo de procesos se evidencia el liderazgo del estratega y el conocimiento 
del entorno, en el segundo grupo salen a flote la racionalidad limitada en la toma de decisiones como consecuencia de información imperfecta, la adaptación y aprendizaje de patrones de comportamiento y la cooperación y la competencia con organizaciones del entorno.

En este orden de ideas, la responsabilidad social con el entorno y la identificación y compromiso de la empresa con los grupos de interés, principios propios de la iniciativa de responsabilidad social empresarial - RSE (Young y Thyil, 2014; Voegtlin y Pless, 2014), se materializan en un modelo de relacionamiento a su vez racional y organizativo.

Este modelo de relacionamiento podría interpretarse como un dispositivo para incentivar a los grupos de interés a colaborar, sabiendo que su comportamiento respondería tanto a estructuras de incentivos como de identidad, basadas estas últimas en la justicia y en la equidad (March et al., 2011).

En tal virtud, el modelo de relacionamiento con grupos de interés se convierte en un instrumento de la responsabilidad social para incorporar la sostenibilidad en la empresa. Se trata de una manera clave de definir reglas de juego entre la empresa y su entorno, y por eso constituye la categoría de análisis del trabajo.

\subsection{Metodología de investigación}

El objetivo es analizar la evolución de la estrategia de responsabilidad social del Grupo EPM entre 2000 y 2013, para esbozar su relación de poder en el área de influencia de la hidroeléctrica Río Grande II, mediante una metodología cualitativa y exploratoria que privilegia la percepción de la empresa, el Estado y la sociedad civil. Se toma como ejemplo esta hidroeléctrica por su importancia para Medellín en cuanto almacena aproximadamente el $36 \%$ del agua que consume esta ciudad.

Se elige el método de caso de Yin (2009), teniendo en cuenta su aplicación en un estudio similar (Liu et al., 2013). Esta elección metodológica es apropiada porque, en palabras de Yin (2009, p. 4),

\begin{abstract}
The more that your questions seek to explain some present circumstance (e.g., "how" or "why" some social phenomenon works), the more that the case study method will be relevant. The method is also relevant the more that your questions require an extensive and "in-depth" description of some social phenomenon.
\end{abstract}

Además, en vista de la poca información existente y del juego de intereses en el área de influencia de la hidroeléctrica, resulta necesario recurrir a entrevistas a diferentes actores y triangular los datos emergentes.

El diseño del caso de estudio exploratorio considera los cinco componentes propuestos por Yin (2009):

1. La pregunta de investigación relativa al adverbio de modo interrogativo "cómo": ¿Cómo define el cambio de estrategia la postura política de la empresa frente al territorio? 
2. El supuesto de investigación de conformidad con el marco teórico: el aprendizaje de la empresa subyacente al cambio de estrategia, contribuiría a la sostenibilidad del negocio de generación de energía si se evidencia una mayor centralidad del Grupo EPM en el relacionamiento con los grupos de interés externos.

3. La unidad de análisis o el caso de estudio: el Grupo EPM y su modelo de relacionamiento con los grupos de interés externo Estado y comunidad.

4. La lógica de asociación entre los datos y el supuesto de investigación: se sigue el supuesto con datos cualitativos tal y como se presenta en el párrafo siguiente.

5. Criterios para interpretar los hallazgos: los resultados pueden interpretarse considerando no solamente la estrategia empresarial como proceso racional y organizativo, sino también otros criterios tales como las tendencias sociales expresadas por los grupos de interés externos. Es decir que, en congruencia con el supuesto de investigación, se examina lo que Yin (2009) denomina societal rival explanations como criterio adicional para interpretar los resultados. Esta estrategia de análisis también es adoptada por Liu et al. (2013) en el caso de la hidroeléctrica de las Tres Gargantas en China, cuando confronta los testimonios del Estado y la empresa en el área de influencia de dicha infraestructura.

Siguiendo este diseño se procede en dos momentos: primero, se estudia el cambio de estrategia, señalando el surgimiento de la responsabilidad social empresarial como hito fundamental. Segundo, se identifica y se discute el efecto político del nuevo modelo de relacionamiento con grupos de interés externos en el territorio influenciado por la hidroeléctrica.

El análisis del cambio de estrategia empresarial se realiza con base en el Plan de manejo ambiental de la hidroeléctrica seleccionada, de los informes de gestión ambiental del grupo empresarial entre 2002 y 2005 , de los informes de gestión ambiental y social entre 2006 y 2008, así como también de los informes de sostenibilidad entre 2009 y 2012.

Esta lectura es confrontada con una selección de entrevistas realizadas a grupos de interés externos, así como a funcionarios del grupo empresarial (cuadro 1). Los grupos de interés externos fueron seleccionados teniendo en cuenta dos criterios. Primero, el nivel de incidencia tanto en la transformación del territorio como en la toma de decisiones a nivel local. Segundo, el nivel de relacionamiento interinstitucional con el Grupo EPM. Por su parte, los funcionarios de la empresa fueron identificados de acuerdo con su nivel de responsabilidad en la concepción y aplicación de la estrategia de responsabilidad social empresarial.

Se privilegiaron entrevistas abiertas y grupos focales con el fin de "indagar un problema y comprenderlo tal y como es conceptualizado e interpretado por los sujetos estudiados, sin imponer categorías preconcebidas" (Bonilla y Rodríguez, 1997, p. 93). Por lo tanto, el entendimiento del proceso racional y organizativo de la estrategia empresarial y de su incidencia política en el territorio es inductivo. 
Asimismo, el análisis del territorio influenciado por la hidroeléctrica Río Grande II se llevó a cabo teniendo en cuenta los resultados más relevantes de la tesis doctoral del autor, actualizándolos con la información secundaria más reciente relativa al manejo de este territorio, es decir, con el nuevo Plan de Manejo del Sistema de Páramos Altoandinos del Noroccidente Antioqueño (Corantioquia, 2010a) y con los Lineamientos de Ordenamiento Territorial para Antioquia (Comisión Tripartita, 2012).

\section{Empresa, territorio y poder: relaciones emergentes con el cambio de estrategia corporativa}

Entre 2000 y 2013 pueden distinguirse dos grandes periodos en la intervención del Grupo EPM en materia social y ambiental. El primer periodo inicia con el establecimiento de la Política Ambiental Corporativa en $2000 \mathrm{y}$, el segundo, con el surgimiento de una nueva estrategia para la sostenibilidad con base en la Responsabilidad Social Empresarial (RSE) en 2006.

Cuadro 1. Interlocutores de la investigación, 2000-2013

\begin{tabular}{|c|c|}
\hline Estado & $\begin{array}{l}\text { Corporación Autónoma Regional del Centro de Antioquia (Corantioquia), autoridad ambiental } \\
\text { - Adolfo Correa, 2013. Coordinador de páramos } \\
\text { - Humberzaro Toro, 2010, 2004, } 2002 \\
\text { - Luis Alfonso Escobar, 2012, 2010, 2004, } 2002 \\
\text { - J. D. Zapata, 2004. Director de Relaciones interinstitucionales } \\
\text { - D. Cardona, 2004. Directora del Banco de proyectos } \\
\text { - M. Arenas, 2002. Directora de la Territorial Tahamíes } \\
\text { - R. Arroyabe, 2002. Funcionario de la territorial Tahamíes } \\
\text { C. E. Orrego, 2002. Funcionaria territorial Hevéxicos } \\
\text { Entidades territoriales } \\
\text { - G. Mejía, 2004. Funcionaria de la Secretaría de Agricultura de la Gobernación de Antioquia } \\
\text { - J. Sierra, 2002. Secretario de la Unidad de Asistencia Técnica Agropecuaria (UMATA) de Don } \\
\text { Matías } \\
\text { - J. Mira, 2002. Funcionario de la UMATA, Don Matías } \\
\text { - Carlos Mario Quiceno, 2002. Secretario del Consejo municipal de Olaya }\end{array}$ \\
\hline $\begin{array}{l}\text { El grupo } \\
\text { EPM }\end{array}$ & $\begin{array}{l}\text { - Carlos Mario Méndez, 2012. Subdirector de Gestión ambiental (hasta 2012) } \\
\text { - Winston Cuellar, 2011. Funcionario Subdirección de Gestión ambiental } \\
\text { - Iván Darío Pineda, 2011. Funcionario Subdirección de Planeación } \\
\text { - Carlos Guillermo Álvarez, 2004. Miembro Junta Directiva (2004-2011) } \\
\text { - Luis Fernando Wolf, 2004. Funcionario de la Subdirección de Planeación } \\
\text { - Melguizo, M., 2004. Unidad de mercado. }\end{array}$ \\
\hline Comunidad & $\begin{array}{l}\text { - Cabildo Verde del municipio de Belmira, Antioquia, organización de la sociedad civil para la pro- } \\
\text { tección ambiental, } 2005 . \\
\text { - Líderes populares del corregimiento de Llanadas en el municipio de Olaya, Antioquia, } 2002 . \\
\text { - C. Londoño, 2004. Director de la unidad de Gestión ambiental desde } 1998 \text { de la Cooperativa } \\
\text { lechera de Antioquia (COLANTA). } \\
\text { - Mauricio Palacio, 2004. Productor de leche en el municipio de Entrerríos. }\end{array}$ \\
\hline
\end{tabular}

Fuente: elaboración propia. 
De este cambio de estrategia parece surgir una nueva postura política de la empresa de cara a su entorno. Es por eso que el análisis de resultados se desarrolla a continuación en dos partes. Siguiendo a Yin (2009), primero se adopta la estrategia Relying on theoretical proposition para analizar el cambio de estrategia a partir de información secundaria. Segundo, se recurre a la estrategia Examining rival explanations para comprender cómo se define la nueva postura política del grupo empresarial frente al territorio, confrontando la percepción de los grupos de interés externos: Estado y Comunidad.

\subsection{Cambio de estrategia corporativa y sostenibilidad del negocio}

Las Empresas Públicas de Medellín (EPM) fueron creadas en 1955, con el fin de asegurar los servicios de agua, electricidad y telefonía a la población de Medellín. Después de 60 años de funcionamiento, las EPM pasan a denominarse Grupo EPM como consecuencia de su proyección nacional e internacional en los negocios de agua, gas natural, energía y saneamiento básico. Este grupo empresarial es de propiedad del municipio de Medellín y está conformado por 45 empresas, de las cuales 20 están en Colombia y 25 de ellas en Centroamérica, el Caribe, Suramérica, Estados Unidos y España (EPM, 2013).

A nivel nacional, su fortaleza se basa en la construcción de grandes obras de infraestructura, en las cuales el Grupo ha venido incorporando progresivamente el manejo socio-ambiental de impactos en el territorio. Este proceso se inicia por mecanismos coercitivos (legislación) y de acceso a créditos internacionales. El Código Nacional de Recursos Naturales (decreto ley 2811 de 1974), la ley 56 de 1981, la ley 99 de 1993 y las condiciones ambientales exigidas por el Banco Interamericano de Desarrollo para acceder a sus créditos lo evidencian, según Carlos Guillermo Álvarez, ex miembro de la Junta Directiva del Grupo EPM. De esta manera se establece en el año 2000 la Política Ambiental Corporativa de EPM (EPM, 2000).

Dicha política se institucionaliza sobre la base de tres principios: el buen uso de los recursos naturales renovables, la planificación y la participación de la población. El buen uso de los recursos naturales renovables reposa sobre el conocimiento de la oferta y la demanda de tales recursos, con el fin de saber administrar los impactos negativos y positivos de los proyectos (EPM, 2005).

La planificación se relaciona con acciones prospectivas que tienden a reducir los costos ambientales (externalidades), privilegiando la investigación, la innovación y la competitividad. La participación de la población pretende tener en cuenta a los clientes y a los trabajadores que intervienen en la prestación de los servicios. Se privilegia la información, la consulta y la concertación entre la empresa y los actores locales.

Entre 2002 y 2005, según los reportes anuales esta empresa ha destinado a la gestión socio-ambiental un promedio anual de aproximadamente USD \$47,2 millones, de los cuales la mayor parte está destinada al buen uso de los recursos naturales renovables, 
el $4,6 \%$ a la participación y el $1 \%$ a la planificación.

En este primer período, la Política Ambiental Corporativa empieza a establecerse al interior y al exterior de la empresa. Al interior, es principalmente la Unidad Estratégica de Negocio de Generación de Energía quien la integra en sus procesos administrativos con un enfoque racional o de planificación. Al exterior, EPM empieza a dibujar el mapa de actores en busca de relacionamientos apropiados con una política que se quiere participativa.

Esta iniciativa de relacionamiento se constituye en el punto de inflexión entre los dos periodos. Entre 2006 y 2013, la Política Ambiental Corporativa se consolida y viene a complementarse en el año 2007 con la nueva Política de Responsabilidad Social Empresarial, que es infundida por la sostenibilidad como una perspectiva más integral de los negocios del grupo empresarial. Así lo establece el Acta 1489 de la Junta Directiva (Gómez Ochoa, 2009; EPM, 2009).

Este cambio de estrategia es voluntario y tiene efecto en la estructura de la organización dado que se crea la nueva gerencia de RSE, como un macro proceso de apoyo a todas las unidades de negocio. Además, se inspira en nuevos estándares internacionales corporativos. Se trata de la voluntad de adhesión al Pacto Global y de contribución al cumplimiento de los Objetivos del Milenio, siguiendo al Global Reporting Initiative (GRI, 2013) y la ISO 26000 (ISO, 2010). De esta manera, la Política de Responsabilidad Social Empresarial (RSE):
... debe ser el marco de actuación dentro del cual se desarrollan los objetivos estratégicos del Grupo Empresarial EPM, para contribuir al desarrollo humano sostenible a través de la generación de valor social, económico y ambiental, acogiendo temáticas sobre sostenibilidad contempladas en iniciativas locales, nacionales y mundiales. La actuación empresarial socialmente responsable tiene un fundamento ético, a partir del reconocimiento de los impactos que sus decisiones tienen sobre los grupos de interés; y se gestionará desde el quehacer diario de la empresa y de las personas que la conforman (EPM, 2009, p. 1).

El principal valor agregado de esta política es el modelo de relacionamiento con los grupos de interés, ya que es sobre este nuevo componente de la estrategia que se sustenta la integralidad de la sostenibilidad del negocio. Desde finales de 2009, dicho modelo se basa en los lineamientos de identificación y relacionamiento del Programa Ambiental de Naciones Unidas (UNEP, 2006). La identificación se lleva a cabo por medio de un triple criterio de legitimidad, urgencia y poder: "Actores sociales con legitimidad, que reciban o puedan recibir impactos de la empresa y al mismo tiempo que tengan capacidad de impactarla" (EPM, 2010, p. 54).

La política de RSE clasifica los grupos de interés en internos, de frontera y externos. Los internos son dueño, socios e inversionistas y servidores. Los de frontera son proveedores y contratistas. Y los externos son clientes y usuarios, comunidad y medio ambiente, competidores y colegas, y el Estado (EPM, 
2012). De este modelo emergen nuevos relacionamientos de la empresa con el territorio impactado por sus activos, principalmente con los grupos de interés externos, pues es allí donde todos interactúan, sea compitiendo por los recursos naturales o tomando decisiones de gestión de los mismos.

Entre los grupos de interés externos, seleccionamos dos debido a su incidencia en las dinámicas territoriales alrededor de la hidroeléctrica Río Grande II. Comunidad y Medio Ambiente es el primero; según el modelo de EPM (2012), está compuesto por la población asentada en las zonas en las cuales el Grupo EPM tiene presencia a través de sus operaciones en la prestación de servicios de energía, agua y gas.

El objetivo de relacionamiento con este grupo es establecer compromisos con las comunidades y otros actores ubicados en las áreas de influencia de los proyectos, obras y actividades en donde la empresa tiene presencia, en relación con la sostenibilidad y la responsabilidad conjunta frente al medio ambiente y al mejoramiento de las condiciones sociales y económicas de dichas comunidades, acorde con las políticas Ambiental y de RSE.

El segundo grupo es el Estado y está conformado por entidades del orden nacional, departamental y municipal, Congreso de la República, entidades reguladoras y de control, autoridades ambientales y entidades internacionales con competencia para regular el funcionamiento de la sociedad y de las empresas prestadoras de servicios públicos.
El objetivo del relacionamiento del Grupo EPM con el Estado es realizar una gestión interinstitucional, de cara a la sostenibilidad ambiental, económica y social del entorno en el que EPM tiene influencia, sin dar lugar a actuaciones que suplanten las funciones de las instituciones estatales.

Como lo expone el ingeniero Carlos Mario Méndez Gallo, ex subdirector ambiental de la Gerencia de Energía del Grupo EPM, este modelo de relacionamiento con el territorio se ve reflejado progresivamente en la gestión ambiental y social de las hidroeléctricas en operación toda vez que se trabaja de manera conjunta, velando por el cumplimiento de las obligaciones legales, la protección de los recursos naturales y la participación activa en el desarrollo.

Es por eso que la corporación parece mostrar una nueva postura frente al manejo del área de influencia de la hidroeléctrica Río Grande II.

\subsection{Una nueva postura política del Grupo EPM frente al territorio}

La lógica de gestión socio-ambiental de las áreas de influencia de las hidroeléctricas del Grupo EPM está relacionada con los períodos mencionados aquí arriba. En un primer momento, la intervención fue impulsada por mecanismos coercitivos $\mathrm{y}$, en un segundo momento, por mecanismos miméticos. En palabras de Perez-Batres et al. (2011), los primeros determinados por la ley y el acceso a recursos y, los segundos, por el comportamiento de las grandes corporaciones internacionales. 
Durante el período de estudio, el perfil de las acciones reportadas con los grupos de interés mencionados en el cuadro 2 contrasta con la evidencia empírica observada en el territorio. Esta evidencia correspondería a lo que Yin (2009) denomina societal rival explanations desde el punto de vista metodológico. En este caso, la evidencia existe desde la fase de construcción y permanece en la fase de operación de la hidroeléctrica.

Desde su construcción en 1989, la hidroeléctrica de Río Grande II parece haberse desarrollado bajo una lógica de enclave económico, toda vez que no se encuentran vínculos claros con el bienestar de la población local. La aplicación de mecanismos obligatorios de compensación como las transferencias del sector eléctrico (ley 99 de 1993), no parecen incidir, entre 1990 y 2010, ni en las condiciones de vida ni en las necesidades básicas de los habitantes de los municipios vecinos al embalse (Agudelo et al., 2012).

Por su parte, la fase de operación se ha caracterizado por múltiples conflictos de interés y acciones interinstitucionales limitadas en el territorio (Polanco, 2013b). Por un lado, el desarrollo agropecuario causa la deforestación y la erosión de los suelos (Polanco, 2011 y 2012). Por el otro, existen títulos mineros que amenazan las fuentes de abastecimiento de agua (Corantioquia, 2010b).

En esta sección, se confrontan las acciones reportadas por el Grupo EPM con las observaciones de campo, para luego evidenciar la nueva postura política del grupo empresarial en el territorio.

\section{Cuadro 2. Acciones del Grupo EPM con grupos de interés externos}

\begin{tabular}{|c|c|}
\hline Comunidad y Medio ambiente & Estado \\
\hline $\begin{array}{l}\text { - Formación y sensibilización } \\
\text { ambiental; } \\
\text { - Atención y seguimiento de la } \\
\text { contaminación hídrica de las } \\
\text { cuencas de los ríos Chico y } \\
\text { Grande, } \\
\text { - Gestión de áreas degradadas } \\
\text { contra la sedimentación del } \\
\text { embalse de Río Grande II, } \\
\text { - Mantenimiento y la conser- } \\
\text { vación de los predios del } \\
\text { embalse (silvicultura), } \\
\text { - Participación en proyectos } \\
\text { que están articulados con } \\
\text { los Planes de Desarrollo y } \\
\text { los Planes de Ordenamiento } \\
\text { Territorial municipal (sanea- } \\
\text { miento básico y vías); } \\
\text { Estructuración, en conjunto } \\
\text { con The Nature Conser- } \\
\text { vancy (TNC), del Fondo del } \\
\text { agua como mecanismo de } \\
\text { inversión en conservación } \\
\text { y restauración de servicios } \\
\text { ambientales (EPM y TNC, } \\
\text { 2011). }\end{array}$ & $\begin{array}{l}\text { - Manejo de resi- } \\
\text { duos de activida- } \\
\text { des agropecua- } \\
\text { rias; } \\
\text { - Control de la } \\
\text { erosión, sanea- } \\
\text { miento básico, } \\
\text { educación am- } \\
\text { biental, produc- } \\
\text { ción más limpia } \\
\text { y reforestación; } \\
\text { Propuesta } \\
\text { normativa en } \\
\text { el marco del } \\
\text { proyecto de ley } \\
\text { 49-10S, también } \\
\text { denominado } \\
\text { como proyecto- } \\
\text { ley de páramos } \\
\text { (2010). }\end{array}$ \\
\hline
\end{tabular}

Fuente: elaborado a partir de los reportes de sostenibilidad del Grupo EPM.

\subsubsection{Relaciones emergentes en el territorio: Grupo EPM, Estado y Comunidad}

Ante los conflictos de interés mencionados anteriormente, las relaciones interinstitucionales pasan a jugar un papel clave. Los actores dominantes en el territorio son el Grupo EPM, la Cooperativa Lechera de Antioquia (Colanta), la autoridad ambiental Corantioquia y las entidades territoriales (municipios de la cuenca de los ríos Chico y Grande y el departamento de Antioquia).

Las organizaciones comunitarias y aquellas no gubernamentales no hacen parte de este 
grupo de actores dominantes ya que están al margen de la toma de decisiones. En la mayoría de los casos (Corporación CEIBA, Cabildo Verde de Belmira y Juntas de Acción Comunal del municipio de Belmira), fungen como operadores de convenios interinstitucionales firmados por los actores dominantes. Según miembros del Cabildo de Belmira y líderes populares del municipio de Olaya, la comunidad local no participa o su participación se confunde con información y formación, sobre todo en temas de protección de fuentes de agua. Es el caso por ejemplo de la formación de gestores ambientes, realizada por la Corporación CEIBA en convenio con Corantioquia.

La importancia del Grupo EPM la determina la presencia de la hidroeléctrica, mientras que la preponderancia de Colanta se debe a su liderazgo en el desarrollo del sector lechero como actividad económica principal del territorio. Corantioquia es la autoridad ambiental y las entidades territoriales se ocupan del desarrollo y el ordenamiento territorial. Las relaciones entre Corantioquia y las entidades territoriales obedecen a una acción normativa de tipo vertical y "directiva", propia de la burocracia de la administración pública, en sentido weberiano (Polanco, 2010).

Además, las entidades territoriales tienen pocos recursos y se tornan dependientes de entidades estatales de orden superior y del sector privado, sobre todo en el caso de los municipios del área de influencia que son todos de categoría V y VI, de acuerdo con la ley 617 de 2000. Así lo expresan los señores Quiceno y Monsalve, Secretario del
Consejo Municipal de Olaya y Director de Planeación de Liborina respectivamente, cuando señalan que el alcance de la gestión municipal es limitado por la racionalización del gasto público que demanda el desempeño fiscal y, por tanto, son pocos los recursos y las voluntades disponibles para llevar a cabo proyectos intermunicipales sin el apoyo de otras organizaciones públicas y privadas.

Por su parte, el relacionamiento entre el Grupo EPM y Colanta es más bien horizontal y de corte "liberal", constituyendo una amenaza para la protección de las fuentes de agua de Río Grande II, pues esta empresa estaría adoptando una actitud pasiva ante el conflicto de intereses.

Esta actitud pasiva de Colanta se infiere del testimonio del ingeniero Londoño, ex director de la Unidad de Gestión ambiental cuando argumenta que las acciones de la empresa se concentran en sus asociados y en el proceso de transformación de leche en la planta del municipio de San Pedro de los Milagros, cerca al embalse. Lo cual desligaría a Colanta de las dinámicas del área de influencia de la hidroeléctrica.

También fue pasivo el relacionamiento entre Corantioquia y el Grupo EPM durante más de diez años, si consideramos la opinión de Humberto Sánchez y Luis Alfonso Escobar, colaboradores de la autoridad ambiental. Sin embargo, se observa que el trabajo mancomunado entre estos actores ha logrado concretarse gracias al convenio interinstitucional 932 de 2013, por medio del cual se destinan aproximadamente USD $\$ 1,18$ millones para desarrollar progra- 
mas de gestión socio-ambiental en áreas de interés común.

Este no es solamente el resultado de múltiples acercamientos, como lo plantea Adolfo Correa, coordinador de páramos de Corantioquia, sino también de la urgencia de solución a problemas comunes como la protección del páramo de Santa Inés que es la fuente de abastecimiento de agua del embalse de Río Grande II y, en gran medida, de la ciudad de Medellín.

\subsubsection{La nueva postura política del Grupo EPM}

De este proceso de relacionamiento del Grupo EPM con el entorno emerge una nueva postura política frente al territorio: parece que la empresa busca obtener una posición más central en el relacionamiento con los actores del territorio. En palabras de Mintzberg (2009), esta centralidad se entiende como el resultado de, por un lado, un mejor conocimiento del entorno que le permite a la empresa tomar decisiones en un contexto de incertidumbre. Por el otro, la centralidad puede obedecer, como lo plantean March et al. (2011) y Prats (2005), al liderazgo del Grupo EPM en el diseño de reglas de juego entre los actores dominantes, que conllevan a una lectura conjunta del territorio.

Como lo exponen Iván Pineda y Jaime Aramburo, colaboradores de la subgerencia de Planeación del Grupo EPM, la postura del Grupo EPM consiste en la necesidad de ir mejorando el relacionamiento con grupos de interés externos para buscar la sostenibilidad de sus proyectos, pero entendiendo que dicho relacionamiento puede o no ser ventajoso dependiendo de las implicaciones para las partes.

Según el Grupo EPM (2010), las oportunidades están en enfoques interinstitucionales de desarrollo regional, que involucren Estado, sector privado y comunidad. Pero el proceso electoral de alcaldes y gobernadores puede afectar la toma de decisiones, puesto que el desarrollo regional es una apuesta a largo plazo que demanda continuidad en las administraciones de estas entidades territoriales. Es por eso que, para el grupo empresarial, el relacionamiento con el Estado pretende bloquear la injerencia política indebida frente a esta continuidad $y$, en cambio, potenciar lo político como constructor de territorio, tal y como se intentó a nivel nacional en el caso del proyecto-ley de páramos en 2010. En palabras de Harrison (2013), lo que trata el grupo empresarial es de señalizar el camino del desarrollo regional, haciéndose indispensable en las redes organizacionales y en los acuerdos de territorio con el Estado a nivel local.

Esta centralidad de EPM con respecto a las entidades territoriales, también existe con Corantioquia. Esto es cierto, por ejemplo, si se acepta la importancia de las relaciones interinstitucionales con la autoridad ambiental en el caso del establecimiento del Fondo del Agua como instrumento de pago por servicios ambientales en el territorio (EPM y TNC, 2011). Aunque el grupo empresarial consultó en un primer momento a Corantioquia sobre la construcción de este instrumento, según Adolfo Correa y Humberto Sánchez, colaboradores de la 
autoridad ambiental, hasta ahora no existe acuerdo entre estos grupos de interés para definir su alcance.

A pesar de que el relacionamiento de EPM con los grupos de interés externos abre lentamente nuevos espacios para acercarse a las necesidades locales, el compromiso que se quiere establecer con las comunidades no es tan claro de cara a las organizaciones productivas locales y su papel en la lectura del territorio enunciada arriba.

Se espera que el mejoramiento de las condiciones sociales y económicas de las comunidades sea el resultado de trabajos conjuntos con organizaciones no gubernamentales y juntas de acción comunal, aunque actualmente funjan como operadores de convenios interinstitucionales entre el Grupo EPM y el Estado. Pero no se espera que dicho mejoramiento sea el resultado de trabajos conjuntos con otros actores ubicados en el área de influencia como las empresas del sector agropecuario, que son los principales agentes del desarrollo económico local.

El principal argumento de esta observación radica en que se trata de nuevos actores con grandes capacidades de transformar el territorio y, por tanto, de incidir indirectamente en la sostenibilidad del negocio de generación de energía. El caso de Colanta es un buen ejemplo. Esta empresa es un vector clave en la propagación de problemas causados por la producción intensiva de leche en la zona de influencia de Río Grande II.

Según el testimonio de Mauricio Palacio, productor lechero de la región, y a los ha- llazgos de Polanco (2011), la deforestación, la erosión de suelos y la contaminación de fuentes hídricas que padecen las cuencas tributarias del embalse, tienen relación con modos extractivos de producción auspiciados por esta Cooperativa. Pero actualmente estas relaciones de causalidad no parecen ser identificadas o, en su defecto, atendidas por los convenios interinstitucionales vigentes.

En definitiva, el Grupo EPM se destaca por la búsqueda de un liderazgo en el desarrollo regional, mientras que el Estado y la comunidad juegan un papel secundario marcado por la falta de recursos y la operación de convenios interinstitucionales respectivamente. La relación de la empresa con estos grupos de interés externos está caracterizada por la gestión del riesgo de generación de energía a través de convenios interinstitucionales con el Estado, y por la información y capacitación de la comunidad. En el otro sentido del relacionamiento, tanto el Estado como la comunidad ven al Grupo EPM como una oportunidad para la consecución de recursos en un contexto de escasez (cuadro 3).

La nueva postura política del Grupo EPM frente al territorio radica en un posicionamiento más central en el mapa de actores, con el fin de hacer del desarrollo regional un proceso compatible con la sostenibilidad de la generación de energía. Este posicionamiento es más claro en relación con el Estado local que en relación con las comunidades locales. La señalización del camino del desarrollo regional por medio de una visión compartida del territorio se evidencia en los acuerdos interinstitucionales. 
Cuadro 3. Matriz de relacionamiento entre actores en el territorio

\begin{tabular}{|c|c|c|c|}
\hline & Grupo EPM & Estado & Comunidad \\
\hline $\begin{array}{l}\text { Grupo EPM } \\
\text { - Negocio de generación } \\
\text { de energía hidroeléctri- } \\
\text { ca }\end{array}$ & $\begin{array}{l}\text { Rol del Grupo EPM: } \\
\text { - Generar energía con } \\
\text { pocos riesgos (sosteni- } \\
\text { bilidad del negocio) } \\
\text { - Liderar el desarrollo re- } \\
\text { gional }\end{array}$ & $\begin{array}{l}\text { Relación del Grupo EPM } \\
\text { con el Estado: } \\
\text { - Celebración de convenios } \\
\text { interinstitucionales como } \\
\text { instrumentos de desarro- } \\
\text { llo regional }\end{array}$ & $\begin{array}{l}\text { Relación del Grupo EPM } \\
\text { con la Comunidad } \\
\text { - Información y forma- } \\
\text { ción }\end{array}$ \\
\hline $\begin{array}{l}\text { Estado } \\
\text { - Corantioquia } \\
\text { - Municipios }\end{array}$ & $\begin{array}{l}\text { Relación del Estado con } \\
\text { el Grupo EPM: } \\
\text { - Consecución de recur- } \\
\text { sos a través de conve- } \\
\text { nios interinstitucionales }\end{array}$ & $\begin{array}{l}\text { Rol del Estado: } \\
\text { - Corantioquia: protección } \\
\text { del páramo de Santa Inés } \\
\text { con pocos recursos } \\
\text { - Municipios: ordenamiento } \\
\text { territorial con limitaciones } \\
\text { presupuestales y de volun- } \\
\text { tad política intermunicipal }\end{array}$ & $\begin{array}{l}\text { Relación del Estado con } \\
\text { la Comunidad } \\
\text { - Información y forma- } \\
\text { ción } \\
\text { - Financiación de pro- } \\
\text { yectos }\end{array}$ \\
\hline $\begin{array}{l}\text { Comunidad } \\
\text { - Organizaciones no gu- } \\
\text { bernamentales (ONG) } \\
\text { - Juntas de acción comu- } \\
\text { nal (JAC) } \\
\text { - Campesinos }\end{array}$ & $\begin{array}{l}\text { Relación de la Comunidad } \\
\text { con el Grupo EPM: } \\
\text { - Solicitud de recursos }\end{array}$ & $\begin{array}{l}\text { Relación de la Comunidad } \\
\text { con el Estado: } \\
\text { - Quejas y reclamos } \\
\text { - Solicitud de bienes y ser- } \\
\text { vicios públicos }\end{array}$ & $\begin{array}{l}\text { Rol de la Comunidad: } \\
\text { - ONG y JAC: Operar } \\
\text { convenios interinstitu- } \\
\text { cionales } \\
\text { - Campesinos: producir } \\
\text { leche para Colanta }\end{array}$ \\
\hline
\end{tabular}

Fuente: elaboración propia.

Sin embargo, quedan dos aspectos por resolver. Por un lado, las comunidades locales siguen estando al margen de estos acuerdos, cuando actúan sobre todo como operadores de los mismos. Por el otro, las actividades agropecuarias organizadas que se desarrollan en el territorio con los pequeños y medianos productores tienen efectos indirectos negativos en la generación de energía. Pero su principal impulsor también está al margen de los procesos de construcción de territorio. Por esta razón sería interesante analizar el relacionamiento entre el Grupo EPM y Colanta.

\section{Conclusión}

La sostenibilidad se incorporó recientemente al Grupo EPM a través de la responsabilidad social empresarial, con un modelo ambicioso de relacionamiento con los grupos de interés. Se trata de un proceso estratégico racional y organizativo, en el sentido de Mintzberg (2009) y Mintzberg et al. (1998), que evidencia un cambio sustancial de las acciones emprendidas por este grupo empresarial para asegurar la sostenibilidad del negocio de generación de energía hidroeléctrica en el área de influencia de los embalses. Este cambio es visible en los documentos institucionales, así como en la percepción de miembros de la Junta Directiva y de colaboradores de la gerencia de energía.

La nueva estrategia de sostenibilidad se plasma en un modelo de relacionamiento del Grupo EPM con sus grupos de interés externos que define una nueva postura política frente al territorio: hacerse más central en el mapa de actores y propender así por la 
compatibilidad del desarrollo regional con la permanencia de sus activos. Como evidencia de ello, se observan dos factores clave: un mejor conocimiento del entorno y el liderazgo en el diseño de reglas de juego para la lectura conjunta y acordada del territorio.

Como lo argumentan Perez-Batres et al. (2011), es la intervención voluntaria que ha hecho efectivo este propósito. En efecto, la construcción colectiva del territorio es, por un lado, una de las principales intenciones promulgadas por el protocolo de sostenibilidad de la International Hydropower Association (2011) y, por el otro, una realidad documentada, aunque en pocos casos, por el programa Dams and Development de la ONU (2007).

No obstante, la construcción colectiva de territorios en países emergentes como Colombia enfrenta dificultades organizacionales en la empresa y las comunidades locales. En la empresa porque todavía predomina un enfoque reactivo ya que existe una tendencia a remediar problemas existentes en lugar de focalizarse en acciones preventivas y de desarrollo local (Valor, 2012).En las comunidades porque prevalece un capital social incipiente. El tejido social, las relaciones de vecindad y la consecuente organización social no tienen peso específico en el juego de actores, dado un relacionamiento más transaccional (comunicación unidireccional, confianza limitada, costos y beneficios diferenciados) que transformacional (comunicación bidireccional, confianza relacional, costos y beneficios compartidos) con agentes dominantes como la empresa (Bowen et al., 2008, Helliwell y Putnam, 2004).
El diseño del modelo de RSE es un hito en el proceso de aprendizaje del Grupo EPM. Queda pendiente consolidar su implementación y construir territorio de cara a procesos transformacionales de desarrollo regional con liderazgo empresarial. Es decir, procesos basados en la comunicación y la confianza en donde la empresa, el Estado y la comunidad compartan costos y beneficios.

Finalmente, el estudio de caso exploratorio desarrollado en este trabajo abre un nuevo panorama investigativo que exige estudios de caso descriptivos y explicativos desde la perspectiva de Yin (2009). En futuras investigaciones valdría la pena analizar si la construcción colectiva del territorio es una práctica común en Colombia, en qué medida está alineada con la estrategia de responsabilidad social empresarial y cómo se relaciona con el ciclo de vida de los proyectos hidroeléctricos: ¿por qué la construcción colectiva del territorio es una práctica que no se piensa desde la fase de factibilidad y se lleva a cabo tanto en la fase de construcción como en la fase de operación de estas obras de infraestructura?, ¿cómo se relaciona todo esto con el desarrollo regional?

Para atender estos interrogantes, el investigador debería ser consciente de la complejidad metodológica puesto que sería necesario abordar técnicas de análisis robustas (ej. Explanation building, Time-Series Analysis) intensivas en información difícil y costosa de producir. 


\section{Referencias}

Agudelo, J. P., Gómez, B. L., y Montoya, J. (2012). Incidencia de la generación de energía en el desarrollo económico local de Antioquia (19902010). Tesis de pregrado en Economía, Universidad de Antioquia, Medellín.

Bernal T., C.A. (2010). Metodología de la investigación: Administración, economía, humanidades $y$ ciencias sociales. Bogotá: Pearson.

Bonilla-Castro, E. y Rodríguez-Sehk, P. (1997). Más allá del dilema de los métodos. La investigación en ciencias sociales. Bogotá: Ediciones Uniandes.

Bowen, F., Newenham-Kahindi, A., and Herremans, I. (2008). Engaging the community: A systematic review. Ontario: Network for Business Sustainability.

Brundtland, G. (1987). Our Common Future. Tokyo: World Commission on Environment and Development.

Chen, M., and Miller, D. (2012). Competitive dynamics: Themes, trends, and a prospective research platform. The Academy of Management Annals, 6 (1), 135-210.

Comisión Tripartita (2012). Lineamientos de ordenamiento territorial para Antioquia. Medellín: Gobernación de Antioquia, Alcaldía de Medellín y Área Metropolitana del Valle de Aburrá. Corporación Autónoma Regional del Centro de Antioquia, Corantioquia (2010a). Plan de manejo del Distrito de Manejo Integrado Sistema de Páramos Altoandinos del Noroccidente Antioqueño. Medellín: Corantioquia.

Corporación Autónoma Regional del Centro de Antioquia, Corantioquia (2010b). Relación de títulos mineros otorgados en el área del DMI Sistema de Páramos y Bosques Altoandinos del Noroccidente Antioqueño. Medellín: Corantioquia.
Dovers, S., and Hezri, A. (2010). Institutions and policy processes: The means to the ends of adaptation. Wiley Interdisciplinary Review - Climate Change, 1 (2), 212-231.

Elden, S. (2010). Land, terrain, territory. Progress in Human Geography, 34 (6), 799-817.

Empresas Públicas de Medellín, EPM (2000). Gestión social integral. Proyecto hidroeléctrico Porce II. Medellín: EPM.

Empresas Públicas de Medellín, EPM (2005). Estados contables, Informes ambientales e Informes de responsabilidad social empresarial 2004. Medellín: EPM.

Empresas Públicas de Medellín, EPM (2009). Política de Responsabilidad Social Empresarial EPM. Medellín.

Empresas Públicas de Medellín, EPM (2010). Informes de Sostenibilidad 2009. Medellín. Recuperado en junio de 2012 de http://www.epm. com.co/site/Home/Institucional/Informesdesostenibilidad.aspx

Empresas Públicas de Medellín, EPM (2012). Informes de Sostenibilidad 2011. Medellín. Recuperado en junio de 2012 de http://www.epm. com.co/site/Home/Institucional/Informesdesostenibilidad.aspx

Empresas Públicas de Medellín, EPM (2013). Historia empresarial del Grupo EPM. Medellín. Recuperado en octubre 2013 de https://www. epm.com.co/site/Home/GrupoEPM.aspx

Empresas Públicas de Medellín, EPM y The Nature Conservancy, TNC (2011). Mecanismos para la conservación y restauración de servicios ambientales en las cuencas abastecedoras de los embalses Riogrande II y La Fe. Manuscrito no publicado.

Fischer, A., Petersen, L., and Feldkoetter, C. (2007). Sustainable governance of natural resources and institutional change, an analytical framework. 
Public Administration and Development, 27 (2), 123-137.

Garriga, E., and Melé, D. (2004). Corporate Social Responsibility Theories: Mapping the Territory. Journal of Business Ethics, 53 (1/2), 51-71.

Global Reporting Initiative, GRI (2013). Sustaina bility Reporting Guidelines \& Technical Protocol G4. Ámsterdam: GRI.

Gómez O., M. (2009). Gestión ambiental en EPM. Documento presentado en CISDA IV, Bogotá, Colombia.

Harrison, J. (2013). Configuring the New 'Regional World': On being caught between Territory and Networks. Regional Studies, 47 (1), 55-74.

Helliwell, J., and Putnam, R. (2004). The social context of well-being. Philosophical Transactions of the Royal Society B, 359, 1435-1446

International Standard Organization, ISO (2010). ISO 26000: Guidance on social responsibility. Geneva: ISO.

Liu, J., Zuo, J., Sun, Z., Zillante, G., and Chen, X. (2013). Sustainability in hydropower development. A case study. Renewable and Sustainable Energy Reviews, 19, 230-237.

Lockwood, M. (2010). Good governance for terrestrial protected areas: A framework, principles and performance outcomes. Journal of Environmental Management, 91 (3), 754-766.

Lozano, R. (2012). Towards better embedding sustainability into companies' systems: An analysis of voluntary corporate initiatives. Journal of Cleaner Production, 25, 14-26.

Lozano, R. (2008). Envisioning sustainability threedimensionally. Journal of Cleaner Production, 16, 1838-1846.

Lozano, R., and Huisingh, D. (2011). Inter-linking issues and dimensions in sustainability reporting. Journal of Cleaner Production, 19, 99-107.

March, J., Friedberg, E., Arellano, D. (2011). Institutions and organizations: Differences and linkages from organization theory. Gestión y Politica Pública, XX (2), 235-246.

Matten, D., and Moon, J. (2008). "Implicit" and "Explicit" CSR: A conceptual framework for a comparative understanding of corporate social responsibility. Academy of Management Review, 33 (2), 404-424.

Mintzberg, H. (2009). Rebuilding companies as communities. Harvard Business Review, JulyAugust.

Mintzberg, H., Lampel, J., and Ahlstrand, B. (1998). Strategy Safari. A guided tour through the wilds of strategic management. New York: The Free Press.

North, D. (1991). Institutions. The Journal of Economic Perspectives, 5 (1), 97-112.

Organization for Economic Co-operation and Development, OCDE (2011). Towards Green Growth: Monitoring Progress. Paris: OCDE.

Pecqueur, B. (2005). Le développement territorial: Une nouvelle approche des processus de développement pour les économies du Sud. In B. Antheaume et F. Giraut (eds.), Le territoire est mort. Vives les territoires! (pp. 295-315). Paris: IRD éditions.

Perez-Batres, L., Miller, V., and Pisani, M. (2011). Institutionalizing sustainability: an empirical study of corporate registration and commitment to the United Nations global compact guidelines. Journal of Cleaner Production, 19, 843-851.

Polanco, J. (2009). Un proyecto de territorio para la protección ambiental: entre discurso global y práctica local. En J. Rosique y A. Gutiérrez (eds.). Estudios sobre territorio, ciudadanía y planeación (pp. 63-92). Medellín: Universidad de Antioquia.

Polanco, J. (2010). Gobernanza del desarrollo económico en el entorno regional de Medellín. Lecturas de Economía, 73, 213-240. 
Polanco, J. (2011). Incidencia del desarrollo socioeconómico en la perennidad del páramo. Gestión y Ambiente, 14 (1), 117-128.

Polanco, J. (2012). Teledetección de la vegetación del páramo de Belmira con imágenes Landsat. DYNA, 79 (171), 222-231.

Polanco, J. (2013a) La incorporación de la sostenibilidad en la empresa de energía, un proceso de aprendizaje técnico y político. Documento presentado en I Simposio sobre Ciencia de la Sostenibilidad, La Habana, Cuba.

Polanco, J. (2013b). La gobernanza del páramo como fuente de abastecimiento de agua. Documento presentado en VI Seminario Internacional de Gestión Ambiental, Medellín, Colombia.

Porter, M. y Kramer, M. (2011). La creación de valor compartido. Harvard Business Review América Latina, Enero.

Prats, J. (2005). De la burocracia al management, del management a la gobernanza. Las transformaciones de las administraciones públicas de nuestro tiempo. Madrid: Instituto Nacional de Administración Pública.

Robinson, J. (2004). Squaring the circle? Some thoughts on the idea of sustainable development. Ecological Economics, 48, 369-384.

Schneider, A., and Meins, E. (2012). Two dimensions of corporate sustainability assessment: Toward a comprehensive framework. Business Strategy and the Environment, 21, 211-222.

Soderbaum, P. (2009). Making actors, paradigms and ideologies visible in governance for sustainability. Sustainable Development, 17 (2), 70-81.

Stagl, S. (2007). Theoretical foundations of learning processes for sustainable development. International Journal of Sustainable Development and World Ecology, 14 (1), 52-62.
United Nations Environmental Program, UNEP (2007). Dams and Development. Relevant Practices for Improved Decision-Making. Nairobi: UNEP Dams and Development Project.

United Nations Environmental Program, UNEP (2006), Accountability, Stakeholder Research Associate. De las palabras a la acción. El compromiso con los stakeholders. Manual para la práctica de las relaciones con los grupos de interés. Nairobi: UNEP.

Valor, C. (2012). The contribution of the energy industry to the millennium development goals: A benchmark study. Journal of Business Ethics, 105, 277-287.

Vazquez-Barquero, A. (2009). Una salida territorial a la crisis. Lecciones de la experiencia latinoamericana. Eure, 35 (105), 5-22.

Vazquez-Barquero, A. (2008). Desarrollo endógeno. Teorías y políticas de desarrollo territorial. Investigaciones regionales, 11, 183-210.

Voegtlin, C., and Pless, N. (2014). Global governance: CSR and the role of the UN global compact. Journal of Business Ethics, 122, 179-191.

Wang, P., Lassoie, J., Dong, S., and Morreale, S. (2013). A framework for social impact analysis of large dams: A case study of cascading dams on the Upper-Mekong River, China. Journal of Environmental Management, 117, 131-140.

Yin, R. (2009). Case study research. Design and methods. Fourth Edition, London: SAGE.

Young, S., and Thyil, V. (2014). Corporate social responsibility and corporate governance: Role of context in international settings. Journal of Business Ethics, 122 (1), 1-24. 
